\title{
A Spinning Reserve Market Considering Security and Biddable Reserve
}

\author{
Haidan Zhao, Kankar Bhattacharya, Senior Member, IEEE, and Jin Zhong, Member, IEEE
}

\begin{abstract}
This paper presents the development of a spinning reserve market model framework for procurement of spinning reserve services, independent of the energy market auctions. The proposed model has developed an innovative spinning reserve biddable reserve calculation scheme which is dynamic and nature, and the full ac OPF is used to determine the spinning reserve procurement while taking into account system security constraints. The model has been applied to the CIGRE 32-bus system and detailed simulation studies have been carried out to examine the performance of the model.
\end{abstract}

Index Terms - ancillary services, electricity markets, optimal power flow, spinning reserve, system security

\section{NOMENCLATURE}

$i, j \quad$ Index for system bus

$k \quad$ Index for hour

$\operatorname{Re}\{I\} \quad$ Real part of transmission line current, p.u.

$\operatorname{Im}\{\mathrm{I}\} \quad$ Imaginary part of transmission line current, p.u.

NG Number of generator buses

NL Number of load buses

MP Energy market price, \$/p.u.MW

$\mathrm{P}_{\mathrm{re}} \quad$ Transmission line real power, p.u.

PD Scheduled real power demand, p.u.

PG Scheduled real power generation, p.u.

$\mathrm{P}^{\mathrm{Max}} \quad$ Maximum capacity of a generator, p.u.

PR Spinning reserve service price offer, \$/p.u.

PSF Energy price scaling factor

QC Reactive power from shunt capacitors, p.u.

Q Transmission line reactive power, p.u.

QD Reactive power demand, p.u.

QG Reactive power generation, p.u.

RAMP Ramp rate of a generator, p.u./h

RSF Reserve price scaling factor

RC Biddable spinning reserve capacity, p.u.

S Complex power, p.u.

This work was partially supported by Natural Sciences and Engineering Research Council (NSERC) of Canada.

Haidan Zhao and Kankar Bhattacharya are with the Department of Electrical and Computer Engineering, University of Waterloo, Waterloo, Ontario, Canada, N2L 3G1.

Jin Zhong is with the Department of Electrical \& Electronic Engineering, The University of Hong Kong, Hong Kong

0-7803-9525-5/06/\$20.00 C2006 IEEE.

$\begin{array}{ll}\mathrm{S}^{\mathrm{Max}} & \text { Maximum limit on complex power, p.u. } \\ \mathrm{SR} & \text { Cleared spinning reserve quantity, p.u. } \\ \mathrm{V} & \text { Magnitude of bus voltage, p.u. } \\ \mathrm{Y} & \text { Magnitude of admittance matrix element, } \text { p.u. } \\ \delta & \text { Angle of bus voltage, radians } \\ \theta & \text { Y-matrix element angle, radians }\end{array}$

II. INTRODUCTION

PINNING reserve is that extra amount of generating capacity, spinning and synchronized with the power system and available for immediate use when system demand increases significantly and suddenly or a contingency (generator or transmission line outage) occurs. Power system operators need to maintain acceptable levels of spinning reserve at all time in order to withstand possible demand excursions or contingencies. Under the traditional regulated utility structure, spinning reserve was determined and allocated during the unit commitment process, while in the new competitive electricity market environment spinning reserve is procured from the market.

Spinning reserve has been defined as an ancillary service in the NERC Operating Manual [1] and has been categorized as a service to maintain a balance between the generation and load. It is classified within a class of contingency reserve services and is required that generators must respond to the ISO requests within 10 minutes. Spinning reserve as an ancillary service in the context of deregulated markets has also been discussed in the research literature. Some proposals have been introduced for the joint scheduling of energy and reserve service [2-7].

A mathematical model as well as its implementation for the New Zealand electricity market has been presented in [2]. The energy supply, energy demand, and reserve are dispatched concurrently in this market with transmission congestion, network losses, reserve requirements, and ramp rate limits considered. The problem is formulated as a linearprogramming model and solved with advanced dual simplex and interior point algorithms. A market model for both power and reserve generation resources was presented in [3]. The dispatch of the resources was constrained by limitations of system transmission capacities.

In [4], a composite market framework for energy and spinning reserve services has been presented. Generators 
submit offers for energy and spinning reserve with ramping limits. Bilateral contracts are also considered in the model with incremental and decremental values specifying the deviation allowed from the schedule, determined by market prices. Transmission security is taken into account using a dc model. The clearing system is formulated as an optimization problem solved using an interior-point method. An energy/reserve joint market considering the reliability of the generators was also proposed by Flynn et al. [5]. It was modeled as an augmented Lagrangian dual function and solved by a recurrent neural network derived from a Hopfield-type network. The price for reserve and the unit reliability are used to find a balance between the cost of reserve and the risk of not providing it.

Wang et al. [6] proposed a joint market for energy and reserve in which energy, spinning reserves and standby reserves are jointly scheduled and dispatched through a mixedinteger model. Arroyo and Galiana [7] presents a contingency and network-constrained market-clearing procedure where energy and spinning reserve are jointly dispatched. It examines the short-term operation and pricing of the various products traded in a joint energy cum reserve market while accounting for transmission network flow limits and security constraints. The optimization process yields the UC solution and the schedules of energy and reserve. It concludes that the requirement and allocation of local reserves are not prespecified but by-products of the market-clearing optimization. The nodal marginal costs of energy and security are also defined. Both generators and loads can submit offers for reserve services in [6] and [7].

The objective of this paper is to establish a spinning reserve service market in deregulated environment wherein the reserve market is separated from the hourly energy market. Such a formulation can be applicable to bilateral contract dominated markets or any mixed form market structure. The proposed market is settled using an optimal power flow framework that considers market constraints and operation constraints while minimizing the total system cost. The selected generators receive a uniform market price of spinning reserve.

\section{DESIGN OF SPINNING RESERVE MARKET}

The proposed market structure for spinning reserve service and the operation time-frame will be described in detail in this section. The market operates in three stages: the first stage involves receiving offers and market settlement, the second stage involves real-time invocation of the service and the third stage is post-operational calculation of payoffs. The work in this paper concentrates on the offer and market settlement process.

The proposed spinning reserve market (SRM) is independent of the energy market and can operate in parallel to the energy market or after the energy market is settled, as shown in Fig. 1 and Fig. 2 respectively. In the case of parallel operation of the two markets, the generators submit their offers one hour before the actual delivery (Fig.1), i.e., they offer at hour $k-1$ for the service delivered from hour $k$ to hour $k+1$.
The market operator settles the energy market and the spinning reserve market simultaneously and determines a uniform market price for the service provision taking into account the system reliability and security constraints and received offers by executing an optimization procedure. The selected generators are activated in real-time after the market settlement.

\section{Energy Market}

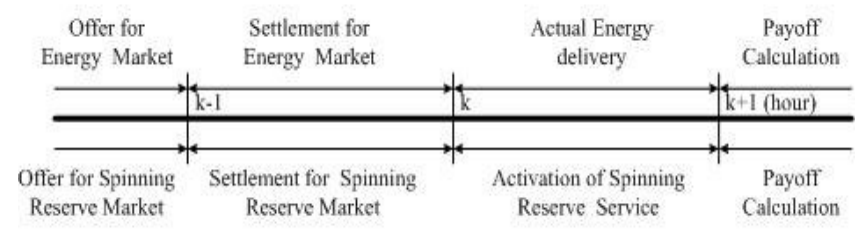

Spinning Reserve Market

Fig. 1. Time-frame of SRM in parallel to energy market

\section{Energy Market}

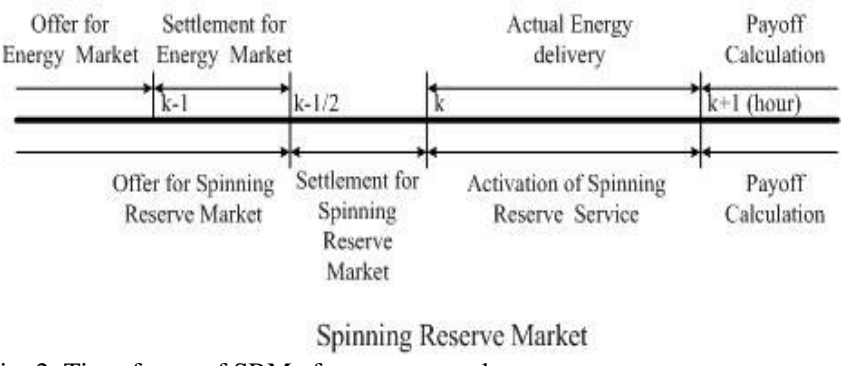

Fig. 2. Time-frame of SRM after energy market

In case of sequential operation of the two markets, the SRM is settled after the energy market settlement (Fig.2). The generators submit their spinning reserve offers half an hour before the actual delivery and after the energy market settlement, i.e., they offer at hour $k-1 / 2$ for the spinning reserve service delivered from hour $k$ to hour $k+1$. The same optimization process is invoked and the market settlement is obtained. The simulations and analytical studies in this paper have assumed the sequential operation of the two markets, although the result can be extended to the simultaneous operation without difficulties.

\section{A. Structure of Offers}

The service providers (generators) submit their offers for spinning reserve services specifying the price and quantity.

The offer price (in \$/MWh) indicates a generator's expectation of price per unit of reserve power for the time period in which the reserve is allocated, but not used. The generator is also paid at the rate of the hourly spot energy price, for the reserve energy that is generated and used. A generator will therefore make profit during the time period, in which the reserve is accepted and used, but incur a revenue loss because of the lost opportunity in the energy market if the spinning reserve is not used. The reserve price is much lower 
than the energy price because the reserve has a lower operating cost.

The offer quantity (in MW) indicates a generator's spinning reserve availability during a given time period. The quantity of spinning reserve which a generator can provide during a onehour period, say, from hour $k$ to hour $k+1$, can be determined from its actual generation at hour $k-1$, its scheduled generation at hour $k$, the ramp rate, and the maximum generation capacity, as shown in Fig.3.

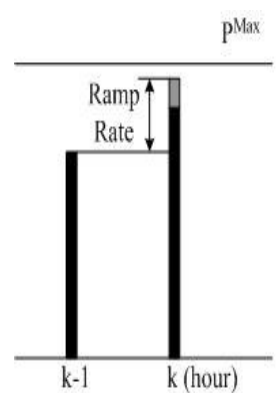

(a)

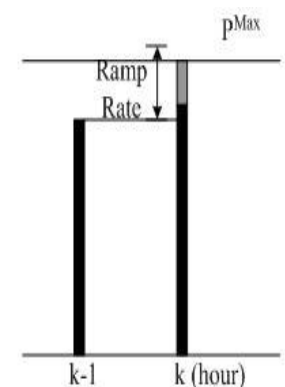

(b)

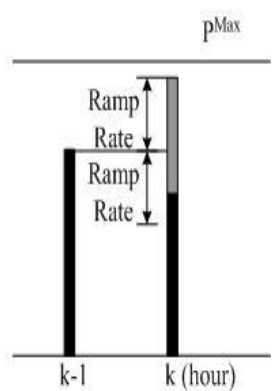

(c)

\section{Scheduled Generation [ Biddable Capacity}

Fig. 3. Evaluation of biddable spinning reserve capacity

As shown in Fig.3 (a), if the scheduled generation for hour $k$ is greater than generation $P G_{k-1}$, then the biddable spinning reserve capacity of a generator at hour $k\left(R C_{k}\right)$ is given as:

$$
R C_{k}=R A M P-\left(P G_{k}-P G_{k-1}\right) \quad \forall P G_{k} \geq P G_{k-1}
$$

In case of Fig.3 (b), the biddable spinning reserve capacity calculated from (1) is limited by the generation capacity and modified as:

$$
R C_{k}=P^{M a x}-P G_{k} \quad \forall P G_{k-1}+R A M P \geq P^{M a x}
$$

If $P G_{k}<P G_{k-1}$, then the biddable spinning reserve capacity is more than the ramp rate, as given below:

$$
R C_{k}=R A M P+\left(P G_{k-1}-P G_{k}\right) \quad \forall P G_{k} \leq P G_{k-1}(3)
$$

To generalize the above conditions, the dynamic biddable spinning reserve capacity of a generator at hour $k$ can be stated using the following relation:

$$
R C_{k}=\left\{\begin{array}{l}
R A M P-\left(P G_{k}-P G_{k-1}\right) \\
\forall P G_{k-1}+R A M P<P^{M a x} \\
P^{M a x}-P G_{k} \\
\forall P G_{k-1}+R A M P \geq P^{M a x}
\end{array}\right.
$$

\section{B. Optimization Model}

Consider a power system with $\mathrm{N}$ buses and $\mathrm{NG}$ generators. Only generators that are participating in the energy market are eligible to submit offers in the SRM. Generator $i$ participates in the SRM by submitting its offer, comprising the price $P R_{i, k}$ and quantity $R C_{i, k}$ for every hour $k$.

The objective of the ISO is to minimize the total cost of procuring spinning reserve services while complying with all market and operation constraints for every hour.

$$
J=\sum_{i}^{N G} P R_{i} \times S R_{i}
$$

In (5), $S R_{i}$ is the cleared spinning reserve. The constraints in this optimization problem can be stated as follows:

\section{1) Bus power balance:}

A full ac-load flow is considered in order to capture the transmission losses accurately and take into account system security constraints such as voltage limits and line-flow (MVA) limits. The generation and load balance for real and reactive power at a bus can be described as follows:

$$
P G_{i}-P D_{i}+S R_{i}=\sum_{j=1}^{N} V_{i} V_{j} Y_{i j} \cos \left(\theta_{i j}+\delta_{j}-\delta_{i}\right)
$$

$$
Q G_{i}-Q D_{i}=-\sum_{j=1}^{N} V_{i} V_{j} Y_{i j} \sin \left(\theta_{i j}+\delta_{j}-\delta_{i}\right)
$$

\section{2) Transmission line limit:}

The transmission line currents are functions of bus voltages and angles, and therefore line real and reactive power flows can be represented using bus voltages and line currents. The transmission line flow is constrained by branch capacity MVA limit. The line flow equations and constraints are given as follows:

$$
\begin{aligned}
& \operatorname{Re}\left\{I_{i j}\right\}=V_{j} Y_{i j} \cos \left(\theta_{i j}+\delta_{j}\right)-V_{i} Y_{i j} \cos \left(\theta_{i j}+\delta_{i}\right)+V_{i} B_{i j} \sin \left(\delta_{i}\right) \\
& \operatorname{Im}\left\{I_{i j}\right\}=V_{j} Y_{i j} \sin \left(\theta_{i j}+\delta_{j}\right)-V_{i} Y_{i j} \sin \left(\theta_{i j}+\delta_{i}\right)+V_{i} B_{i j} \cos \left(\delta_{i}\right) \\
& \left.P_{i j}=V_{i} \mid \operatorname{Re}\left\{I_{i j}\right\} \cos \left(\delta_{i}\right)+\operatorname{Im}\left\{I_{i j}\right\} \sin \left(\delta_{i}\right)\right] \\
& \left.Q_{i j}=V_{i} \mid \operatorname{Re}\left\{I_{i j}\right\} \sin \left(\delta_{i}\right)-\operatorname{Im}\left\{I_{i j}\right\} \cos \left(\delta_{i}\right)\right] \\
& S_{i j}=\sqrt{P_{i j}^{2}+Q_{i j}^{2}} \\
& S_{i, j} \leq S_{i j}^{M a x}
\end{aligned}
$$

\section{3) Reserve limits:}

The reserve that can be procured by the ISO and cleared in the market is limited by the reserve offer quantity, i.e., the biddable reserve capacity, from generators.

$$
0 \leq S R_{i} \leq R C_{i} \forall i=1, \ldots, N G
$$


Where $R C_{i}$ is given by:

$$
R C_{i, k}=\left\{\begin{array}{r}
R A M P_{i}-\left(P G_{i, k}-P G_{i, k-1}\right) \\
\forall \quad P G_{i, k-1}+R A M P_{i}<P_{i}^{M a x} \\
P_{i}^{M a x}-P G_{i, k} \\
\forall \quad P G_{i, k-1}+R A M P_{i} \geq P_{i}^{M a x}
\end{array}\right.
$$

\section{4) Contingency constraint:}

Spinning reserve is often required to be a certain percentage of the load or be capable of making up the loss of the largest generator while sometimes these reserve requirements have been calculated as a function of the probability of not having sufficient generation to meet the load. The contingency constraint helps examine various load deviation conditions and contingencies to arrive at suitable reserve procurements. For example, when generator $i$ is considered to be on outage, the constraint can be described as $P G_{i}=0$ and $R C_{i}=0$.

\section{5) Generator reactive power limits}

These constraints ensure that the reactive power provided by the generators is within their reactive capacity limits.

$$
Q G_{i}^{\min } \leq Q G_{i} \leq Q G_{i}^{\max } \quad \forall i=1, \ldots, N G
$$

\section{6) Bus voltage limits}

The voltage of load buses should be maintained at an acceptable level to ensure the power quality.

$$
V_{i}^{\text {Min }} \leq V_{i} \leq V_{i}^{\text {Max }} \quad \forall i=1, \ldots, N L
$$

The generator bus voltages are maintained at constant levels.

$$
V_{i}=\bar{V}_{i} \quad \forall i=1, \ldots, N G
$$

\section{7) Market Price and Payment}

The uniform market price is determined from the highest offer selected by the market operator after the optimization process. All selected spinning reserve providers receive this uniform market price for the reserve that is allocated but not used. The generators also receive the energy price for the reserve generated if the reserves are activated in real-time. The payments are calculated after real-time according to the uniform market price and the energy price.

\section{CASE STUdY}

A modified version of the IEEE 30-bus system is used to demonstrate the functioning of the proposed SRM for various cases. There are 30 buses in the system, of which 6 buses are generator buses. The transmission system includes nine 132 $\mathrm{kV}$ buses, while the distribution system includes twenty-one 33 $\mathrm{kV}$ buses. The transmission system and the distribution system are interconnected with 4 transformers.

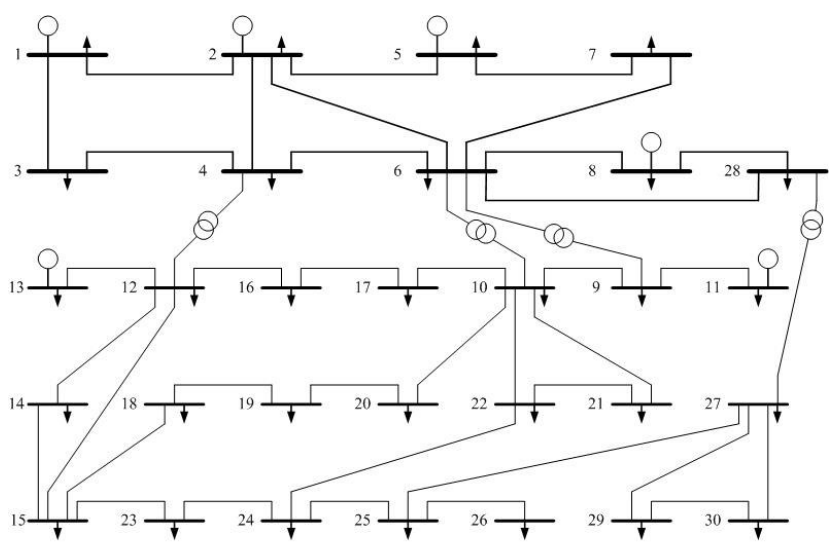

Fig. 4. Modified IEEE 30-Bus system

There may be various strategies adopted by a competitive generator to formulate its price offer for the spinning reserve service. In this work, the price offer is constructed as a function of the energy demand and market price.

$$
P R_{i}=P S F \times R S F \times \text { uniform }\left(\text { Price }^{\text {Min }}, \text { Price }^{\text {Max }}\right)
$$

In (19), $P S F$ is a price scaling factor that is related to the chronological energy demand for the day. The cost of providing spinning reserve service is high when the demand is high, and vice versa. RSF is a scaling factor for the reserve price vis-à-vis the expected energy market price. The uniform function provides a uniform random number generation in the range of the expected energy market price for the generator. This is used to model the offer price structure in the absence of more realistic price forecast information.

The spinning reserve quantity offer of a generator can be determined by calculating the hourly biddable reserve capacity. The market operator uses the SRM settlement model described in Section-2 to obtain the optimal spinning reserve allocation schedule and the uniform market price.

Consider a spinning reserve requirement to meet an unexpected 10\% increase in demand. Fig.4 shows the relationship of total capacity, demand/generation, biddable reserve capacity and cleared reserve in the system. The total capacity is 10 p.u. in the system. The peak loads occurs during hours $9-13$ and hours $17-21$. It can be observed that the cleared reserve is the highest during these periods. However, the biddable reserve capacity is lowest during these hours because of the high demand. It is observed from Fig.4 that at some hours the margin between the biddable reserve capacity and the cleared reserve is quite small. This might give rise to gaming situation in the system by some imperfect market participants (generators). 


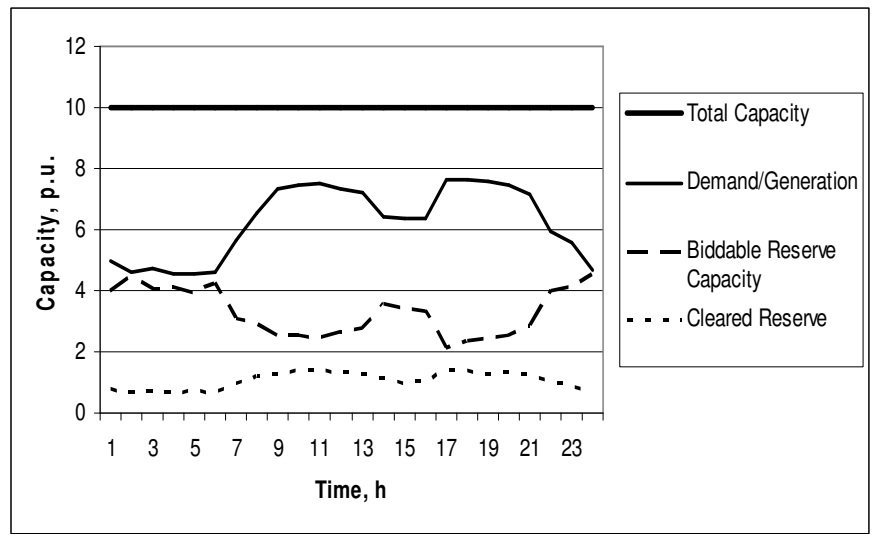

Fig. 5. Relationship of total capacity, demand/generation, biddable reserve capacity and cleared reserve in the system

The spare capacity in the system is calculated from the difference between the total capacity and the total generation.

\section{Spare Capacity $=$ Total Capacity - Total Generation}

A comparison of spare capacity and biddable reserve capacity (Fig.6) shows the diminishing difference between the two, during hours 9-18, which can be a cause for concern.

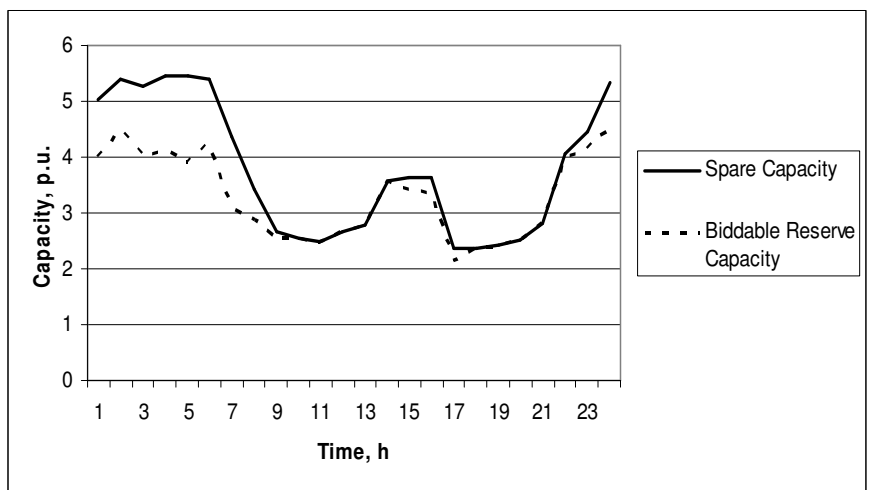

Fig. 6. Comparison of spare capacity and biddable reserve capacity

The biddable reserve capacity in the system over 24 hours vis-à-vis the spinning reserve market prices are shown in Fig.7. It can be observed that the spinning reserve price increases significantly when the biddable reserve capacity is low. The peak price during a day is about three times of the off-peak price.

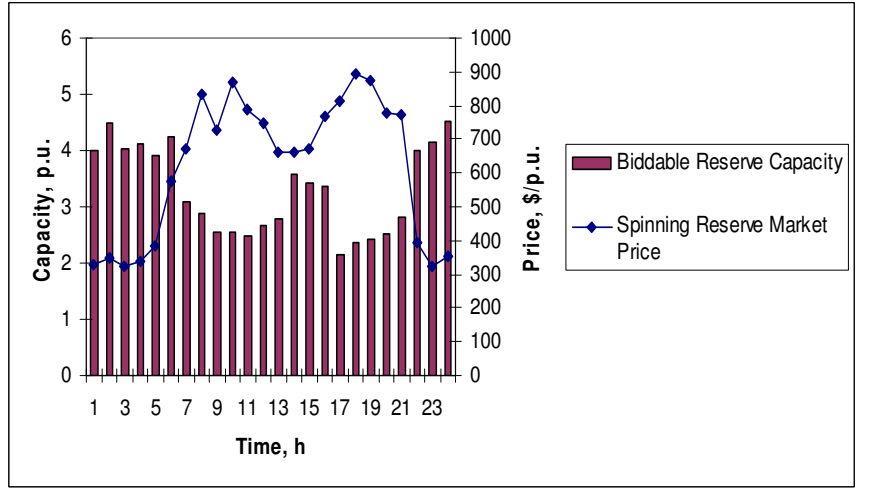

Fig. 7. Spinning reserve market price vis-à-vis biddable reserve capacity
The SRM price may increase when the transmission line limit is taken into consideration. If the transmission line limit is reduced and reaches a certain minimum level, the system is not able to survive because it is not able to procure enough effective spinning reserve through the transmission network. The system can survive under different transmission line limit at different load demand levels.

Figure 8 shows the minimum feasible transmission line limits at each hour, for which the system can operate safely. The corresponding spinning reserve market prices are also shown in the same figure. It is observed that when the transmission constraints are more binding (i.e., the minimum feasible line limit is high) the spinning reserve market prices are high, and vice versa.

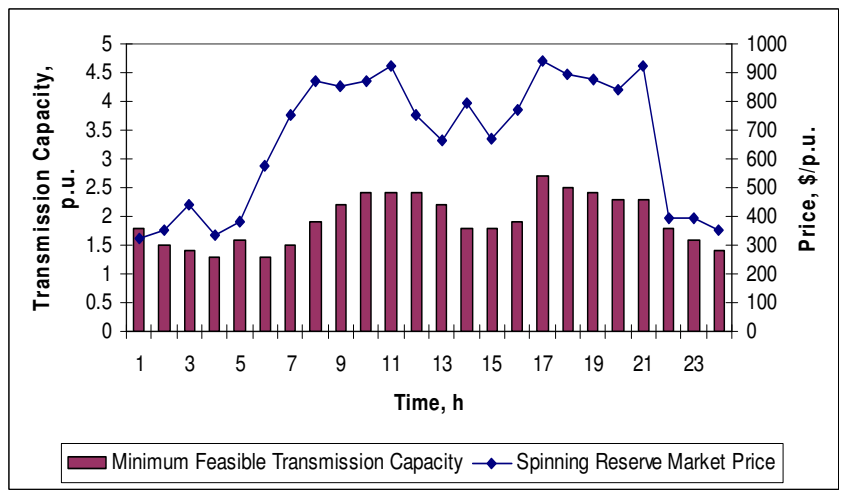

Fig. 8. Spinning reserve market price vis-à-vis transmission line flow level

Figure 9 shows a comparison, with and without maximum transmission line limits. It is observed that the prices at hour 17 and hour 21 increase when line flow limits are imposed. The market operator is forced to accept higher price offers because of constraints on the line flow, which results in the increase of market price.

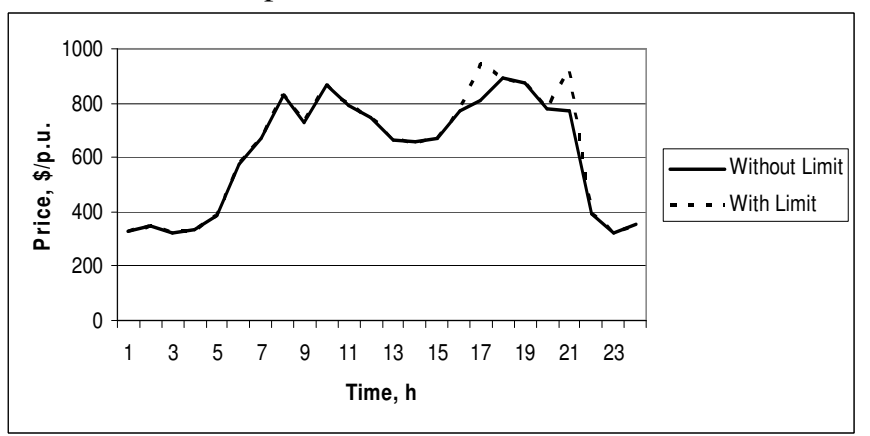

Fig. 9. Comparison of SRM price with and without maximum transmission line flow limits

Figure 10 shows a comparison of SRM price with and without the minimum feasible transmission line limit. It is observed that the market prices with limits are generally higher than those without limits. 


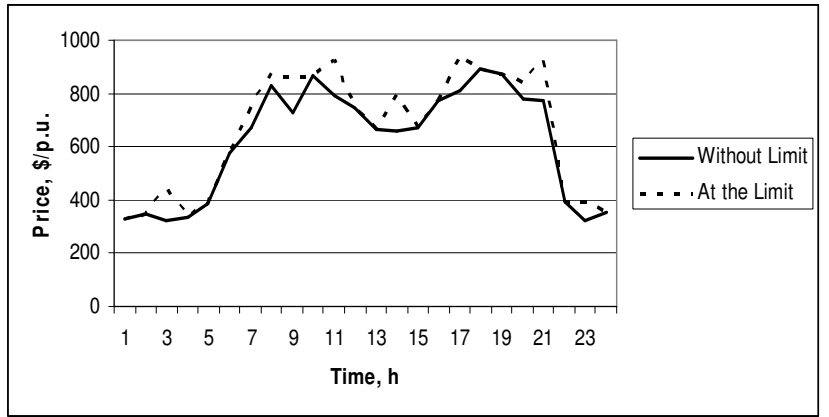

Fig. 10. Comparison of SRM price with and without minimum feasible transmission line flow limit

Consider an outage of the generator at Bus 1 (capacity = $150 \mathrm{MW}$ ). It is observed from Fig.11 that the biddable reserve capacity decreases and the SRM price increases significantly. Further considering a case when transmission line 12-16 is on outage, we observe that the SRM price increases during some hours (Fig.12).

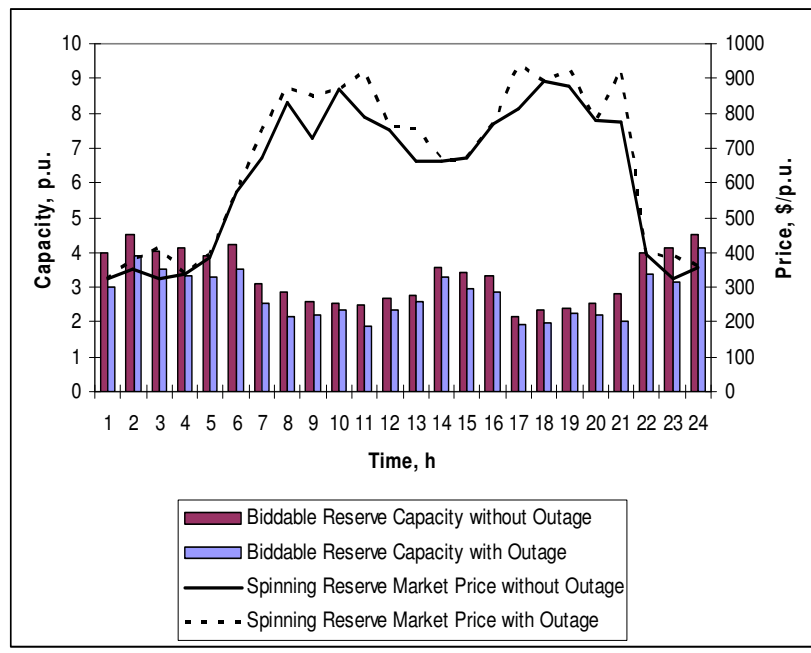

Fig. 11. Comparison of biddable reserve capacity with and without generator outage and corresponding SRM price

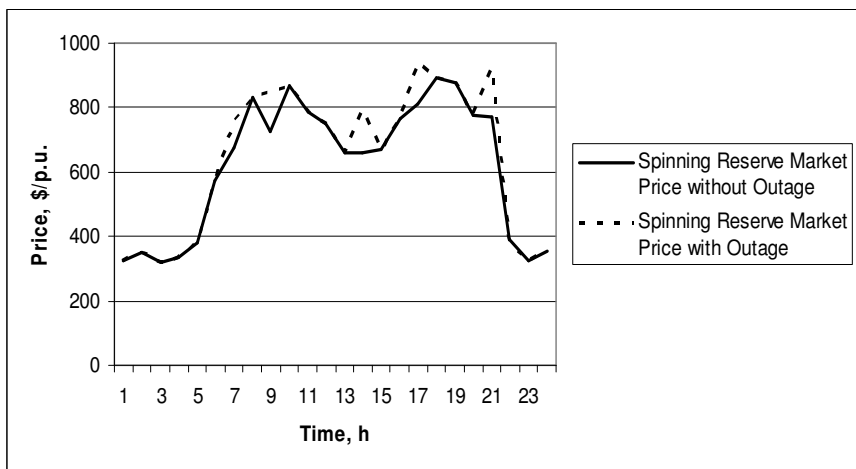

Fig. 12. Comparison of SRM price with and without transmission line outage

The corresponding SRM prices are also determined. It is observed that when transmission constraints are more binding (i.e., the minimum feasible line limit is high) the SRM prices are high, and vice versa. Table-1 shows the cleared spinning reserve services during the peak load hour (hour 17) for each generator in the various cases discussed earlier. It is observed that the spinning reserve offer from Gen-2 is not accepted in the base case (without transmission line limits and outages). When transmission line flow constraints are applied or there are outages, the market operator is forced to accept the more expensive offer from Gen-2, hence the market price increases.

TABLE-1: ClEARED SPINNING RESERVES (IN P.U.MW) IN VARIOUS CASES

\begin{tabular}{|c|c|c|c|c|}
\hline Generator & $\begin{array}{c}\text { Base } \\
\text { Case }\end{array}$ & $\begin{array}{c}\text { With } \\
\text { Transmission } \\
\text { Line Limit }\end{array}$ & $\begin{array}{c}\text { With } \\
\text { Generator } \\
\text { Outage }\end{array}$ & $\begin{array}{c}\text { With Line } \\
\text { Outage }\end{array}$ \\
\hline Gen-1 & 0.23 & 0.23 & 0 & 0.23 \\
\hline Gen-2 & 0 & 0.14 & 0.43 & 0.47 \\
\hline Gen-5 & 0.43 & 0.39 & 0.65 & 0 \\
\hline Gen-8 & 0.05 & 0.05 & 0.05 & 0.05 \\
\hline Gen-11 & 0.1 & 0.1 & 0.1 & 0.1 \\
\hline Gen-13 & 0.61 & 0.5 & 0.61 & 0.61 \\
\hline
\end{tabular}

\section{CONCLUSIONS}

This paper establishes a spinning reserve service market model, which is separated from the energy market. The offer from a generator comprises a price offer and quantity offer that is the biddable capacity considering the scheduled generation and ramp rate. The proposed market is settled using an optimal power flow model that considers market constraints and operational constraints including transmission line constraints, while minimizing the total system cost. The selected generators receive a uniform market price of spinning reserve. Simulation is carried out on the IEEE 30-bus system. Finally, case studies considering transmission line flow limit, generator outage and transmission line outage are conducted.

\section{REFERENCES}

[1] North American Electric Reliability Council, "NERC Operating Manual". June 2004.

[2] T. Alvey, D. Goodwin, X. Ma, D. Streiffert, and D. Sun, "A securityconstrained bid-clearing system for the New Zealand wholesale electricity market," IEEE Trans. Power Systems, vol. 13, pp. 340-346, May 1998.

[3] M. Aganagic, K. H. Abdul-Rahman, and J. G. Waight, "Spot pricing of capacities for generation and transmission of reserve in an extended Poolco model," IEEE Trans. Power Systems, vol. 13, pp. 1128-1135, Aug. 1998.

[4] M. Madrigal, and V. H. Quintana, "A security-constrained energy and spinning reserve markets clearing system using an interior-point method," IEEE Trans. Power Systems, vol. 15, pp. 1410-1416, Nov. 2000.

[5] M. Flynn, W. P. Sheridan, J. D. Dillon and M. J. O'Malley, "Reliability and reserve in competitive electricity market scheduling," IEEE Trans. Power Systems, vol. 16, pp. 78-87, Feb. 2001.

[6] J. Wang, N. E. Redondo, and F. D. Galiana, "Demand-side reserve offers in joint energy/reserve electricity markets," IEEE Trans. Power Systems, vol. 18, pp. 1300-1306, Nov. 2003.

[7] J. M. Arroyo, and F. D. Galiana, "Energy and reserve pricing in security and network-constrained electricity markets," IEEE Trans. Power Systems, vol. 20, pp. 634-643, May 2005.

\section{BIOGRAPHIES}

Haidan Zhao obtained her Bachelor and Master degrees from South China University of Technology, China, in 1994 and 2001 respectively. Thereafter, she worked for the Southern China Architectural Design Institute, Haikou, China during 1994-1998 and 
the Guangzhou Building Design Institute, Guangzhou, China during 2001-2002. Thereafter, she obtained her MASc from University of Waterloo, Waterloo, Canada in 2005.

Kankar Bhattacharya (M'95, SM'01) received the $\mathrm{Ph} . \mathrm{D}$. degree in electrical engineering from Indian Institute of Technology, New Delhi, in 1993. He was with the Faculty of Indira Gandhi Institute of Development Research, Bombay, India, during 1993-1998, and the Department of Electric Power Engineering, Chalmers University of Technology, Gothenburg, Sweden, during 1998-2004. Since January 2003, he has been with the Department of Electrical and Computer Engineering, University of Waterloo, Canada, as an Associate Professor. His research interests are in power system dynamics, stability and control, economic operations planning, electricity pricing and electric utility deregulation. Dr. Bhattacharya received the 2001 Gunnar Engström Foundation Prize from ABB Sweden for his work on power system economics and deregulation issues.
Jin Zhong (S’00, M'05) received the B.Sc. (Eng.) degree from Tsinghua University, Beijing, China, in 1995 and the M.Sc. (Eng.) degree from the Electric Power Research Institute, Beijing, in 1998, and the Ph.D. degree from Chalmers University of Technology, Gothenburg, Sweden, in 2003. She worked as a Researcher at the Electric Power Research Institute, Beijing, during 1998-1999. In 2002, she spent five months as a Visiting Scholar at Washington State University, Pullman, USA. Currently, she is an Assistant Professor in the Department of Electrical and Electronic Engineering at the University of Hong Kong. Her areas of interest are electricity sector deregulation and ancillary service pricing. 